\title{
Optimizing system capacity expansion schedules for groundwater supply
}

\author{
Liang-Cheng Chang, ${ }^{1}$ Yu-Wen Chen, ${ }^{1}$ and Ming-Sheng Yeh $^{1}$ \\ Received 7 January 2008; revised 9 February 2009; accepted 20 April 2009; published 10 July 2009.
}

[1] Groundwater plays a vital role in regional water resources management. In conventional design, a full-scale network system is installed initially to use groundwater resources. However, the system capacity may exceed water demand in the early stages because water demand generally increases with time. Therefore, this work proposes a novel optimal capacity expansion model capable of determining an optimal schedule to expand system capacity according to increasing water demand. The proposed algorithm hybridizes a genetic algorithm (GA) and constrained differential dynamic programming (CDDP). The chromosomes of the GA represent a possible design alternative, a groundwater network with capacity that expands with time. The CDDP algorithm is then used to compute the optimal pumping policy associated with the chromosome. Simulation results indicate that the capacity expansion model saves more total present value cost than conventional designs for the same annual interest rate and water demand. Results of this study demonstrate promise for the proposed model in facilitating a cost-effective groundwater network design with capacity expansion for regional groundwater supply.

Citation: Chang, L.-C., Y.-W. Chen, and M.-S. Yeh (2009), Optimizing system capacity expansion schedules for groundwater supply, Water Resour. Res., 45, W07407, doi:10.1029/2008WR006835.

\section{Introduction}

[2] Water demand increases with time because of increasing population and rising economies. Incrementally expanding water resource capacity is a cost-effective strategy that satisfies increasing water demand [Braga et al., 1985; Rosegrant and Cai, 2002; Jenkins et al., 2004; PulidoVelazquez et al., 2006]. Optimizing capacity expansion has become a significant issue in water resources management [Voivontas et al., 2003; Mahmoud, 2006]. However, previous investigations cannot effectively solve the groundwater capacity expansion problem under fully dynamic conditions. Developing an optimal groundwater capacity expansion model that can determine an optimal network expansion schedule and consider time-varying pumping rates to fulfill increasing water demand is an important issue.

[3] Voivontas et al. [2003] and Mahmoud [2006] studied optimal capacity expansion for a surface water system. Voivontas et al. [2003] developed a capacity expansion model of an integrated water resource management for islands. Their model considers the conjunctive operation and expansion schedule of an integrated water resources system including groundwater wells, reservoirs, desalination plants and shipping water. Mahmoud [2006] applies an optimization model to determine the optimal expansion schedules of a desalination plant. Mahmoud integrates

\footnotetext{
${ }^{1}$ Department of Civil Engineering, National Chiao Tung University, Hsinchu, Taiwan.
}

Copyright 2009 by the American Geophysical Union. 0043-1397/09/2008WR006835 objective space dynamic programming (OSDP) and mixed integer programming to solve the capacity expansion problem. Both studies focus on the expansion schedule of surface water and either ignore or greatly simplify groundwater systems as a subsurface reservoir without considering the dynamic hydraulic head.

[4] For a capacity expansion problem that emphasizes the groundwater system, the decision variables are mainly the well network and its expansion schedule that include the location and installation schedule of each well in the design network. Furthermore, for the groundwater capacity expansion problem, computing the spatially distributed hydraulic head is necessary, requiring much more computational work than a problem considering only the surface water system. Başağaoğlu and Yazicigil [1994] propose capacity expansion models to determine the optimal expansion schedules for a hypothetical multiaquifer system using mixed integer programming and the response matrix method. Their study examines the impact of various demand increasing curves, including the linear curve, convex curve and concave curve, and simplifies operation cost to planning a capacity expansion system. The study indicates that simplified operation cost affects computational time and total cost, and demand requirement variations may influence variations in selecting well network design and expansion schedule. Watkins and McKinney [1998] developed a capacity expansion model for conjunctive use management. Their model also applies the response matrix method to simulate hydraulic head response for a confined aquifer. The model computes the optimal network design and associated expansion schedule by assuming steady pumping rates. Both studies determine the optimal network design and associated expansion schedule, and simplify the capacity expansion problem by as- 
suming steady pumping rates or linear operation cost. The studies also apply the response matrix method to simulate hydraulic head response that implies a linear assumption for the groundwater system.

[5] For a groundwater system planning problem that considers a time-varying pumping system, the hydraulic head will vary in time and become a dynamic optimization problem. The problem progresses to a nonlinear dynamic problem when considering a nonlinear objective function or nonlinear groundwater system such as an unconfined aquifer. Because water demand increases with time, time-varying pumping rates are more cost-effective than constant pumping. However, solving a nonlinear dynamic optimization problem is computationally demanding and previous studies propose methods to increase computational efficiency [Hsiao and Chang, 2002; Chang and Hsiao, 2002; Chu et al., 2005; Hsiao and Chang, 2005; Chang et al., 2007]. Hsiao and Chang [2002] have further designed optimal well networks with time-varying pumping rates. They apply a hybrid algorithm, genetic algorithm (GA) and constrained differential dynamic programming (CDDP) to solve the discrete nonlinear dynamic optimization problem. Their study simultaneously determines optimal well network designs and a set of time-varying pumping rates. Using the CDDP algorithm, the proposed model computes timevarying pumping rates with sixty periods for a 15-year planning horizon. They further apply the hybrid algorithm to groundwater remediation planning problems [Chang and Hsiao, 2002; Chu et al., 2005; Hsiao and Chang, 2005; Chang et al., 2007].

[6] To summarize the previous discussion, for a groundwater system planning problem, expanding system capacity according to increasing water demand and considering timevarying pumping rates is the most cost effective system planning strategy. However, the problem will be a discrete nonlinear dynamic problem without significant simplification and solving the problem is computationally demanding. Very few methodologies proposed by previous studies can solve the problem efficiently. Although Hsiao and Chang [2002] propose a model to solve a discrete nonlinear dynamic groundwater planning problem, their network system design is static and does not expand with schedule. Their methodology cannot directly apply to a groundwater capacity expansion problem without modification. Therefore, this study proposes a novel methodology to solve a groundwater system planning problem considering capacity expansion by integrating GA and the modified CDDP, a hybrid procedure customized from Chang et al.'s studies [Hsiao and Chang, 2002; Chang and Hsiao, 2002; Chu et al., 2005; Hsiao and Chang, 2005; Chang et al., 2007]. The algorithm optimizes the capacity expansion problem on an unconfined aquifer while minimizing total costs, including fixed costs and operation costs.

\section{Formulation}

\subsection{Formulation of the Capacity Expansion Problem}

[7] The objective function of the optimal planning model minimizes the present total cost value. The total cost consists of fixed cost for well network installation and well pumping operation cost. Mathematical formulations of the optimization model, including the objective function and required constraints, are as follows:

$$
\begin{aligned}
\min _{\left\{I, u_{i, t}\right\}} z= & \sum_{i \in I}\left\{c_{1} y_{i} \frac{1}{(1+R)^{n\left(P_{i}-1\right)}}+\sum_{t=1}^{T}\right. \\
& \left.\cdot\left[c_{2} u_{i, t}\left(L_{i}-h_{i, t+1}\right) \frac{1}{(1+R)^{t}}\right]\right\}, I \subset \Omega
\end{aligned}
$$

subject to

$$
\begin{gathered}
\frac{\partial}{\partial x}\left(K_{x x} \cdot h \frac{\partial h}{\partial x}\right)+\frac{\partial}{\partial y}\left(K_{y y} \cdot h \frac{\partial h}{\partial y}\right) \\
+\sum_{i \in \Omega} u_{i} \delta\left(x_{i}, y_{i}\right)=S_{y} \frac{\partial h}{\partial t} \\
t=1,2, \ldots, T \quad i \in I \\
h_{i, t+1} \geq h_{\min }, \quad t=1,2, \ldots, T \quad i \in I \\
\sum_{i \in I} u_{i, t} \geq d_{t}, \quad t=1,2, \ldots, T \quad i \in I \\
u_{\min } \leq u_{i, t} \leq u_{\max }, \quad t=1,2, \ldots, T \quad i \in I
\end{gathered}
$$

where

$\Omega$ is a set of the candidate locations to install a well network within the aquifer;

$I$ is a set of well locations and is also a subset of $\Omega$;

$u_{i, t}$ is the decision variable, time-varying pumping rate, for the $i$ th well at the $t$ th operation time step;

$i$ denotes the $i$ th well within the candidate network I;

$t$ denotes the $t$ th operation time step;

$c_{1}$ is the fixed cost of well installation for unit length;

$c_{2}$ is the unit cost of pumping water and also can be formulated as $c_{2}=\rho \times g \times c_{3} \times \Delta t$;

$c_{3}$ is the cost of electric power per unit work;

$\rho$ is water density;

$g$ is acceleration due to gravity;

$y_{i}$ is the depth of the $i$ th well in the candidate network;

$P_{i} \quad$ is the construction period of the $i$ th well and each construction period contains $n$ operation time steps $(n \geq 1)$;

$R$ is the interest rate;

$h_{t+1}$ is the hydraulic head at operation time step $t+1$;

$L_{i}$ is the ground surface elevation for the $i$ th well;

$d_{t}$ is the amount of the water demand at operation time step $t$;

$u_{\text {min }}$ is the minimum pumping rate;

$u_{\max }$ is the maximum pumping rate;

$h_{\text {min }}$ is the required minimum groundwater head.

Equation (1) represents the present total cost value. The first term of equation (1) is the present fixed cost value and the second term is the operation cost. The decision variable is $u_{t, i}$, which is the pumping rate for the $i$ th well in the candidate network at time step t. Equation (2) is the transfer function of hydraulic head for an unconfined aquifer. The associated numerical model for equation (2) is modified 


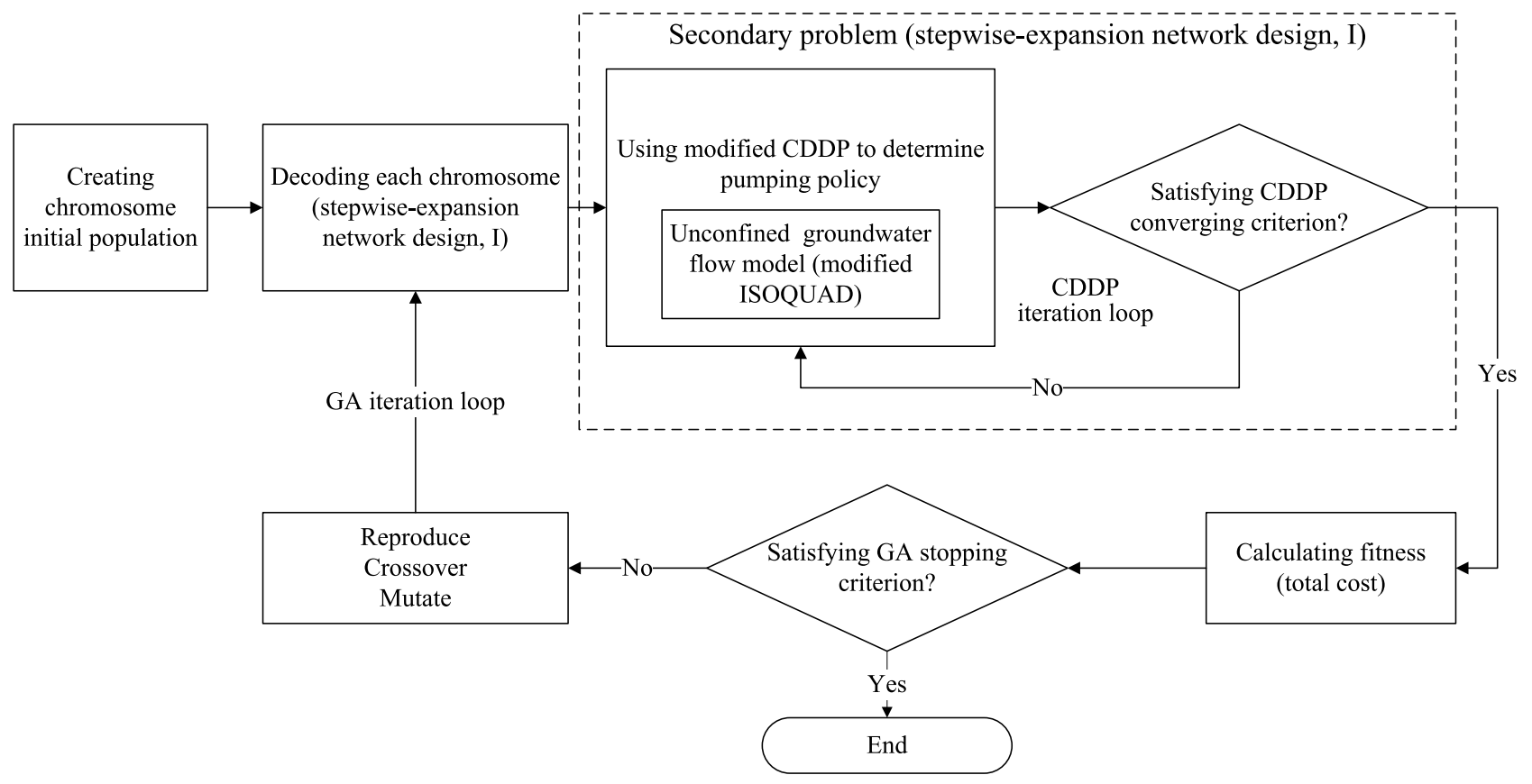

Figure 1. Hybrid algorithm of the groundwater capacity expansion planning model.

from ISOQUAD [Pinder and Frind, 1972], which is a finite element numerical model originally designed for a confined aquifer. The thickness of an unconfined aquifer varies with the groundwater table over time; therefore this work applies the Picard method to update aquifer thickness value in the ISOQUAD modification. Equation (3) ensures that the optimal pumping policy does not cause environmental impact by groundwater overpumping. Equation (4) ensures that system productivity fulfills the water demand for each time step. Equation (5) is the capacity constraint for each well.

[8] The proposed groundwater optimal planning problem as defined by equations (1)-(5) is a mixed discrete, nonlinear, and dynamic optimization problem. The decision variables include discrete variables describing the well network designs, and continuous variables describing the time-varying pumping rates. The capacity and number of wells in each network design increase at a specific time step. Conventional gradient-based algorithms, such as nonlinear programming (NLP), cannot solve this problem because of discrete variables. The number of continuous decision variables, i.e., time-varying pumping rates, also increase with each time step. Computational loading greatly increases with increasing the number of time steps when using only conventional mixed nonlinear programming (MNLP) or other heuristic algorithms such as the genetic algorithm (GA), taboo search (TS), or simulation annealing (SA). No single algorithm adequately solves the problem defined by equations (1)-(5). Therefore this study proposes a hybrid algorithm.

\subsection{Hybrid Algorithm to Solve the Groundwater Capacity Expansion Problem}

[9] This section describes the hybrid algorithm modified from studies by Hsiao and Chang [2002], Chang and Hsiao [2002], Chu et al. [2005], Hsiao and Chang [2005], and Chang et al. [2007]. The algorithm development includes two steps, "problem reformulation" and "GACDDP integration."

\subsubsection{Reformulation of the Problem}

[10] This study explores the problem structure and reformulates the problem into a two-level optimization problem to facilitate hybrid algorithm development and ensure that the algorithm consistently solves the problem defined by equations (1)-(5). The two-level optimization problem is formulated asPrimary problem

$$
\min _{\{I\}} z=\sum_{i \in I}\left(c_{1} y_{i} \frac{1}{(1+R)^{n\left(P_{i}-1\right)}}\right)+J^{*}(I), I \subset \Omega
$$

Secondary problem (for each network alternative design, I)

$$
J^{*}(I)=\min _{\left\{u_{i, t}\right\}} \sum_{t=1}^{T} L_{t}=\min _{\left\{u_{i, t}\right\}} \sum_{t=1}^{T} \sum_{i \in I} c_{2} u_{i, t}\left(L_{i}-h_{i, t+1}\right) \frac{1}{(1+R)^{t}}
$$

subject to equations (2), (3), (4), and (5).

[11] Solving the two-level optimization problem defined by equations (6) and (7) and associated constraints (equations (2)-(5)) is equivalent to solving the original one defined by equations (1)-(5). However, the two-level formulation decomposes the original problem into two groups. The primary problem contains all the discrete variables: the fixed cost and the discrete decision variables, stepwise expansion network designs. This study uses a GA to solve the primary problem. Each chromosome (stepwise expansion network design) within the GA is associated with a secondary problem as indicated by equation (7) and the secondary problem solution is the time-varying pumping rate for the network design. The secondary problem contains all nonlinear and dynamic characteristics. The objective function (equation (7)) and constraints (equations (2)- 




$\square$ Potential well

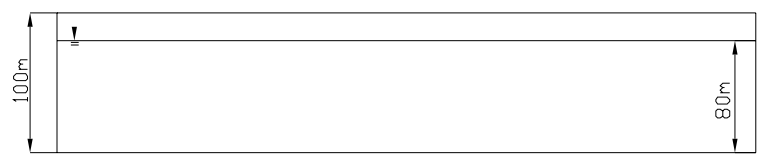

Figure 2. Aquifer used for case study (modified from Chang et al. [1992]).

(5)) in the secondary problem are nonlinear and are separable in time. Hence, the CDDP algorithm, a nonlinear dynamic algorithm, is suitable to solve the secondary problem.

\subsubsection{GA-CDDP Integration}

[12] This study develops a hybrid algorithm by integrating the GA and modified CDDP as illustrated in Figure 1, to solve the two-level formulation described in equations (2)(7). The hybrid algorithm in Figure 1 includes two parts: GA iteration and modified CDDP. The GA accomplishes the discrete search for an optimal stepwise expansion network design. This work uses the modified CDDP algorithm during the GA search to determine the optimal timevarying pumping rates for each well in the network design by minimizing the present value of operation cost. The main hybrid algorithm, as indicated in Figure 1, is a simple GA embedded in a modified CDDP algorithm. The whole algorithm solves the primary problem defined by equation (6) and the CDDP solves the secondary problem defined by equation (7) associated with each chromosome.

[13] Although Hsiao and Chang [2002], Chang and Hsiao [2002], Chu et al. [2005], Hsiao and Chang [2005], and Chang et al. [2007] provide the basic principle for the hybrid GA-CDDP, their work requires significant modifications. The modification involves two important issues: one is chromosome coding of the stepwise expansion network design, and the other is CDDP modification to adapt the stepwise expansion network.

[14] First, the chromosome-coding scheme used by the GA indicates how each chromosome represents a stepwise expansion network design. A binary string composed of binary bits represents the chromosome. This study assumes three expansion intervals for the pumping network, therefore four options of well installation exist for each candidate well site; not installing the well, installing the well at the beginning of interval $\mathrm{I}$, installing the well at the beginning of interval II, and installing the well at the beginning of interval III. Two binary bits are used to encode the four options, creating a chromosome of twice as many bits as the total number of candidate wells. Each two-bit binary is decimalized into 0 to 3 . The decimalized value that equals 0 indicates no well will be installed at the associated candidate site; otherwise, the decimalized value that equals 1,2 or 3 represents a pumping well will be installed at the associated candidate site in the beginning of interval I, II or III.

[15] Second, the CDDP algorithm modification is needed because the number of decision variables increase with expansion intervals. Since pumping rates for each well are the decision variables in the CDDP algorithm, the number of wells for each network design also defines the number of decision variables. For a stepwise expansion network, pumping wells are installed at the beginning of each expansion interval. Since the network designs (chromosomes) have defined the number of wells at each interval, CDDP modification is possible. For each time step, a second-order Taylor's expansion (shown as equation (8)) is used to approximate the operation cost (equation (7)) on the nominal policy [Murray and Yakowitz, 1979; Jones et al., 1987]:

$$
\widetilde{L}_{t}=\delta x_{t}^{T} \widetilde{A}_{t} \delta x_{t}+\delta u_{t}^{T} \widetilde{B}_{t} \delta x_{t}+\delta u_{t}^{T} \widetilde{C}_{t} \delta u_{t}+\widetilde{D}_{t} \delta u_{t}+\widetilde{E}_{t} \delta x_{t}
$$

Equation (8) contains five coefficient matrixes, decision variables $\left(u_{t}\right)$ and state variables $\left(x_{t}\right)$. The variable $u_{t}$ is a $m \times 1$ vector, the variable $x_{t}$ is a $n \times 1$ vector, where $\mathrm{m}$ is the number of decision variables and $\mathrm{n}$ is the number of state variables. $\widetilde{A}_{t}$ is a $n \times n$ matrix, $\widetilde{B}_{t}$ is a $m \times n$ matrix, $\widetilde{C}_{t}$ is a $m \times m$ matrix, $\widetilde{D}_{t}$ is a $m \times 1$ vector and $\widetilde{E}_{t}$ is a $n \times$ 1 vector. The number of decision variables, according to the previous discussion, increase at the beginning of each expansion interval; hence the size of the coefficient matrix $\left(\widetilde{B}_{t}, \widetilde{C}_{t}, \widetilde{D}_{t}\right)$ also varies accordingly. Since the chromosomes specify the number of decision variables and expansion schedule before CDDP computation, the CDDP algorithm can be modified to adapt the time-varying size of decision variables and coefficient matrix $\left(\widetilde{B}_{t}, \widetilde{C}_{t}, \widetilde{D}_{t}\right)$.

\subsubsection{Stopping Criterion for GA-CDDP}

[16] The proposed algorithm is composed of the GA and the CDDP; therefore, the stopping criterions of both parts should be defined specifically. The stopping criterion of the GA is that the optimal fitness value is the same for 10 consecutive generations. The stopping criterion of CDDP is that the ratio of objective function value change between two consecutive iterations is less than a constant value, $\varepsilon_{J}$ (shown as equation (9)):

$$
\left|J^{(n+1)}-J^{(n)}\right| /_{J^{(n)} \leq \varepsilon J}
$$

\section{Numerical Results}

[17] The current work performs numerical studies on the basis of a hypothetical groundwater problem modified from Chang et al. [1992] that verifies effectiveness of the proposed approach. This work assumes the aquifer (Figure 2) of the hypothetical problem as homogeneous and isotropic, and uses the modified ISOQUAD to simulate groundwater variation. The site area is $3000 \times 5000 \mathrm{~m}^{2}$ and described with 77 finite element nodes, sixty elements, and 


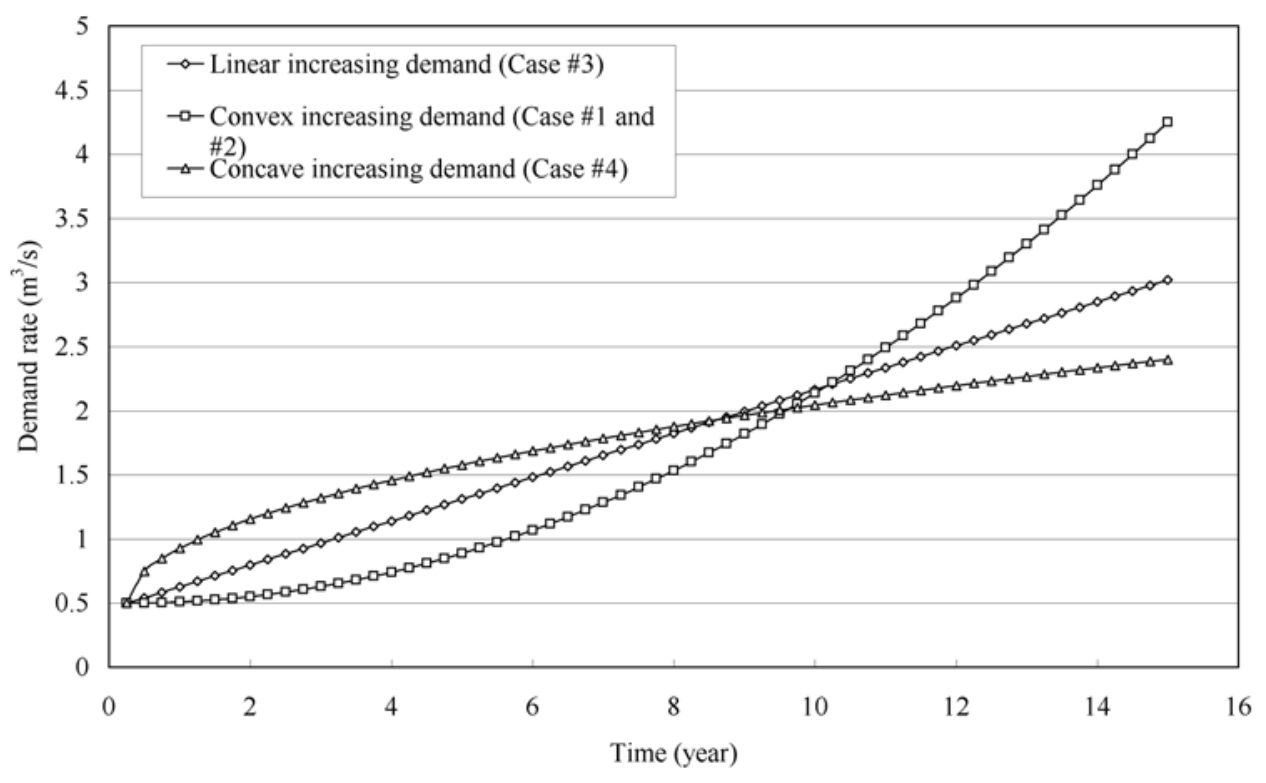

Figure 3. Water demand curves used in this study.

35 candidate well locations. Constant head and no-flow boundaries circumvent the flow domain. Initial conditions on hydraulic head for each node are assumed to be $80 \mathrm{~m}$ and the aquifer thickness is $100 \mathrm{~m}$. Table 1 lists aquifer properties and simulation parameters. The entire planning horizon in the management model is 15 years and is divided into three capacity expansion intervals and a total of sixty simulation time steps. The simulation time step is 91.25 days (equals a quarter of year). Each interval contains twenty simulation time steps and each interval is 5 years. Wells are installed at the beginning of each interval. This study investigates three different demand curves including the convex curve, linear curve and concave curve [Başă̆aoğlu and Yazicigil, 1994], as Figure 3 illustrates. The following demonstrates four cases. Case 1 is a nonexpansion case associated with the convex curve of demand increasing. Cases 2, 3, and 4 are expansion cases, where demand increases on the basis of a convex, linear and concave demand curve.

[18] Parameters related to the GA, including population size, crossover probability, and mutation probability, are determined by GA parameter sensitivity analysis. Population size and crossover probability for performing GA sensitivity analysis ranged from 80 to 120 and 0.5 to 0.8 , respectively. The mutation probability for each sensitivity computation is assigned as the multiplicative inverse of population size [De Jong, 1975]. Population size, crossover probability, and mutation probability were specified as 100 , 0.8 and 0.01 , respectively, on the basis of sensitivity analysis (shown as Table 1).

\subsection{Total Cost Saving of Capacity Expansion}

[19] Results of cases 1 and 2 shown in Table 2 illustrate total cost saving caused by employing the capacity expansion design concept. Case 1 is a nonexpansion (NE) case and case 2 is a capacity expansion (CE) case. Both case 1 and case 2 apply convex demand curves. The optimal construction policy in case 1 installs 22 wells during initial planning, while optimal solution for case 2 also installs 22 wells but with the policy to install 5,6 , and 11 wells at intervals I, II and III, respectively. The fixed present value cost for cases 1 and 2 are $\$ 440,000(100 \%)$ and $\$ 280,392$ (64\%) USD, respectively. Therefore, a capacity expansion model saves $36 \%$ of fixed cost compared to a nonexpansion model. The operation present value cost for cases 1 and 2 are $\$ 992,484(100 \%)$ and $\$ 993,005(100.05 \%)$ and the total present value cost is $\$ 1,432,484(100 \%)$ and $\$ 1,273,398$ $(89 \%)$. The result clearly indicates that with only a slight increase of operation cost $(0.05 \%$ more $)$, the capacity expansion model saves $11 \%$ of total cost for the hypothet-

Table 1. Aquifer Properties, Simulation, and Optimization Parameters of Example Application

\begin{tabular}{lc}
\hline & Value \\
\hline Aquifer properties & \\
Specific yield & 0.1 \\
Porosity & 0.2 \\
Horizontal and vertical & $0.005 \mathrm{~m} / \mathrm{s}$ \\
$\quad$ hydraulic conductivity & \\
Initial groundwater head & $80 \mathrm{~m}$ \\
$\quad$ Aquifer thickness & $100 \mathrm{~m}$ \\
Simulation parameters & \\
$\quad$ Simulation time step length & $(\mathrm{D}), 0.25(\mathrm{Y})$ \\
$\quad$ Capacity expansion interval length & $5(\mathrm{Y})$ \\
$\quad$ Planning horizon & $15(\mathrm{Y})$ \\
$\quad$ Electric power cost & $0.45^{\mathrm{a}}$ \\
$\quad$ (dollars per kilowatt hour) & $200^{\mathrm{a}}$ \\
$\quad$ Fixed cost for unit length & \\
$\quad$ well installation & \\
Optimization parameters & 0.01 \\
Mutation probability for GA & 10 \\
Number of generations for GA & \\
$\quad$ stopping criterion & $10^{-5}$ \\
$\quad$ Maximum absolute changing rate for & \\
$\quad$ CDDP stopping criterion & 100 \\
Population size for GA & 0.8 \\
Crossover probability for GA &
\end{tabular}

${ }^{a}$ Values are in U.S. dollars. 
Table 2. Summary of Numerical Results Under Different Design Policies and Different Water Demand Curve Types ${ }^{\mathrm{a}}$

\begin{tabular}{|c|c|c|c|c|}
\hline & \multirow{2}{*}{$\frac{\text { Nonexpansion Model }}{\text { Case } 1}$} & \multicolumn{3}{|c|}{ Capacity Expansion Model } \\
\hline & & Case 2 & Case 3 & Case 4 \\
\hline Water demand curve type & convex & convex & linear & concave \\
\hline Number of wells for period 1 & 22 & 5 & 7 & 8 \\
\hline Number of wells for period 2 & 22 & 11 & 11 & 11 \\
\hline Number of wells for period 3 & 22 & 22 & 16 & 13 \\
\hline Present value of fixed cost (USD) & 440,000 & 280,392 & 239,126 & 218,494 \\
\hline Present value of operation cost (USD) & 992,484 & 993,005 & $1,059,023$ & $1,123,846$ \\
\hline Present value of total cost (USD) & $1,432,484$ & $1,273,398$ & $1,298,149$ & $1,342,340$ \\
\hline
\end{tabular}

${ }^{\mathrm{a}}$ The cost of unit length well installation $\left(c_{1}\right)$ is $\$ 200$ U.S. dollars (USD). Average water demand for 15 years $\left(\mathrm{m}^{3} / \mathrm{s}\right)$ is 105.65 . The annual interest rate is $8 \%$.

ical case. Fixed cost reduction results in total present value cost savings for the capacity expansion model. Although the number of installed wells for both cases is the same, installing part of the wells required for the future saves the total present value of fixed cost because of interest saving.

[20] A look into the efficiency of system capacity utilization further explores cost saving of the capacity expansion design. Case 1 requires first fulfilling maximum water demand which only occurs at the end of the planning horizon, and most system capacity is unused at intervals I and II, during the first 10 years, as shown in Figure 4. However, the cost (the interest) for unused capacity must still be paid. The designed pumping system capacity for the capacity expansion case (case 2) requires water demand satisfaction at each interval; therefore, the unused capacities are much less than that of case 1. The shadow area in Figure 4 is the amount of unused capacity saved by the capacity expansion model.

[21] Figures 5, 6, 7, and 8 illustrate optimal pumping well locations and water levels at the end of the planning horizon for all cases. The water levels represent various policy influences on the groundwater system. The water level value and pattern shown in Figure 5 and 6 for cases 1 and 2 , respectively, are very similar. Results are consistent with the assumption; water demands are the same for the two cases, hence, groundwater pumping and water tables are also expected to be similar. On the other hand, water demand at the end for case 3 is larger than that of case 4 according to Figure 3. Therefore, pumping at the end for case 3 should be larger than that for case 4 and water levels for case 3 are expected to be less than for case 4 . Water levels demonstrated in Figures 7 and 8 fulfill the expectation. Results indicated from Figures 5-8 demonstrate that the proposed model optimizes a groundwater capacity expansion problem as defined in equation (2).

\subsection{Impact of Varied Increasing Water Demand Rate on Present Value Cost}

[22] The impacts of varied increasing water demand rate on present value cost can be illustrated by examining the results of cases 2, 3, and 4 summarized in Table 2. Although total demands for the three cases are the same and average water demand is $105.65 \mathrm{~m}^{3} / \mathrm{s}$, the types of water demand

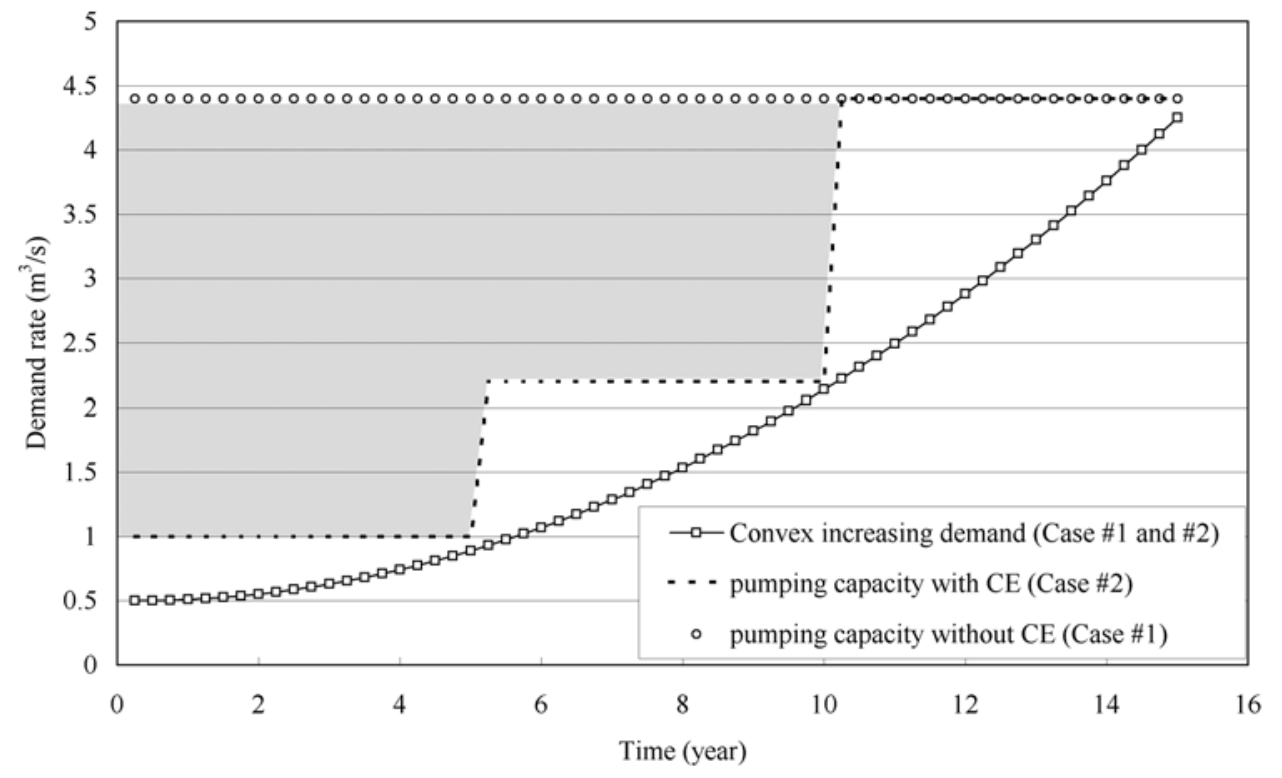

Figure 4. The time-varying total pumping capacities with and without considering capacity expansion. 
$\boldsymbol{\Delta}$ : wells for period I, $\boldsymbol{\bullet}$ :wells for period II, $\bullet$ :wells for period III

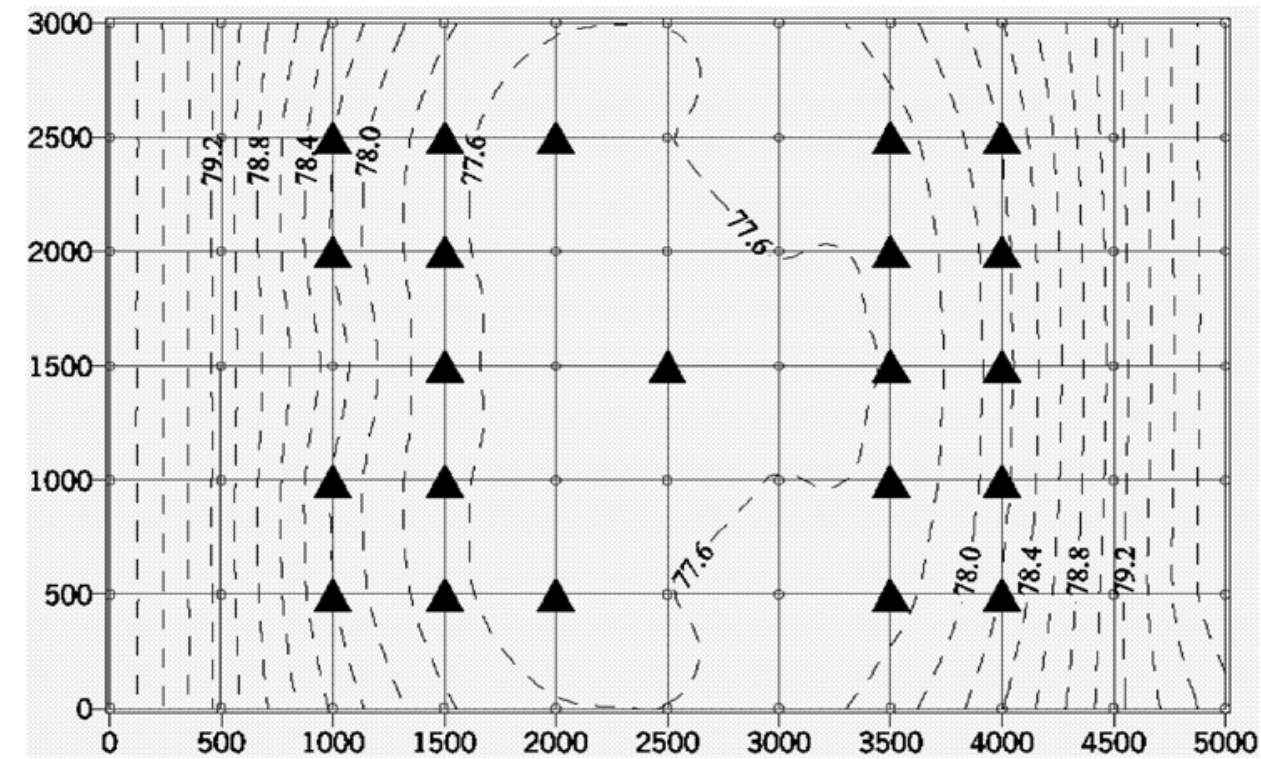

Figure 5. The water level of case 1 (NE and convex increasing demand) at the end of the 15 th year.

increase for cases 2, 3, and 4 are different, namely, the convex curve, linear curve, and concave curve, as Figure 3 demonstrates. Water demands increase with time, as do system capacities indicated in Table 2 . In case 2 , the number of wells in the pumping system for the optimal expansion schedule at the three expansion intervals are 5, 11, and 22. The numbers of wells for case 3 are 7, 11, and 16, and 8, 11, and 13 for case 4 . Water demand quantity also defines the number of required wells because of each well capacity constraint. Therefore, case 2 has the greatest number of wells at the end since it has the largest water demand at the ending period as shown in Figure 3. Case 2's numbers of wells at the ending period are much larger than those of cases 3 and 4; therefore, case 2 has the greatest fixed cost. The present value of fixed costs for cases 2, 3, and 4 are $\$ 280,392$ (100\%), \$239,126 (85\%), and \$218,494 (78\%), respectively. Equation (1) indicates that the more the pumping volume, the higher the operation cost. Therefore, the operation cost distribution in time is similar to their demand curves, and case 2 will have a higher operation cost than the other case near the end of the planning period. However, because of the discounting effect of computing present

\section{$\boldsymbol{\Delta}$ : wells for period I, $\boldsymbol{a}$ :wells for period II, $\bullet$ :wells for period III}

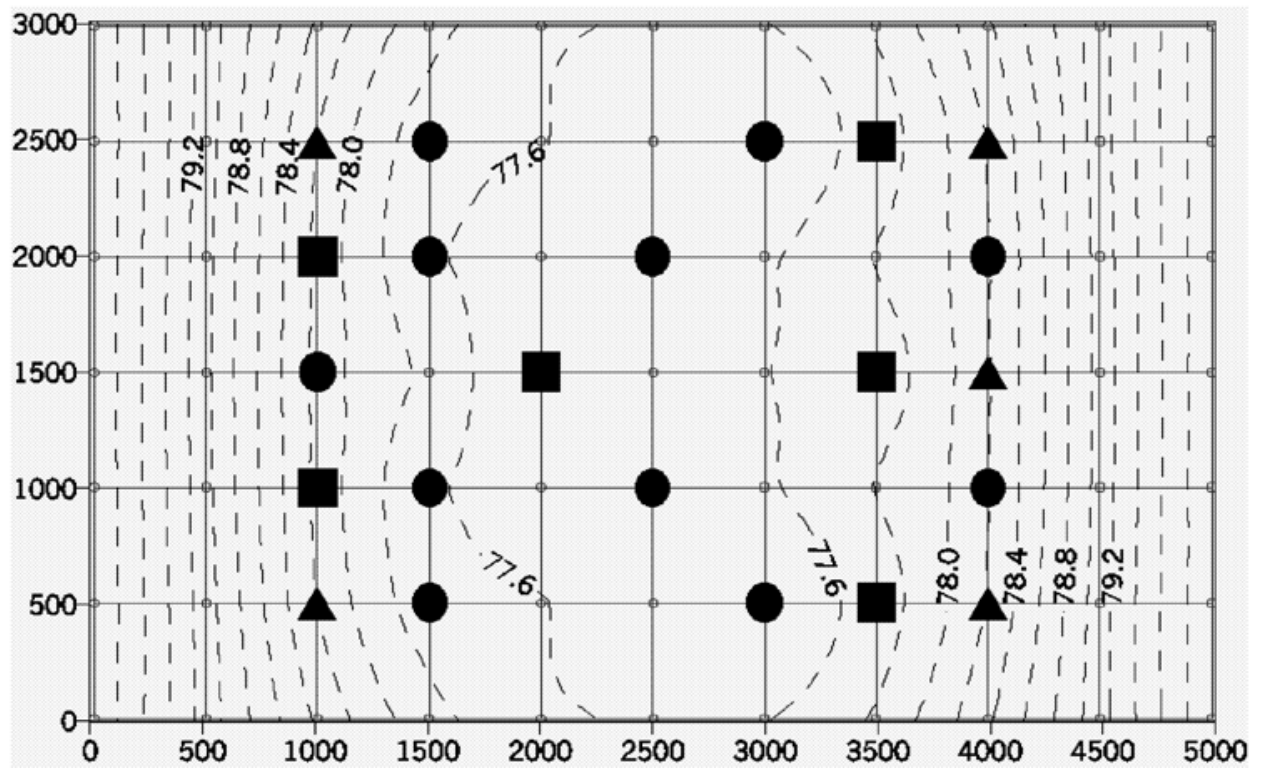

Figure 6. The water level of case 2 (CE and convex increasing demand) at the end of the 15 th year. 
$\boldsymbol{\Delta}$ : wells for period I, $\boldsymbol{\bullet}$ :wells for period II, $\bullet$ :wells for period III

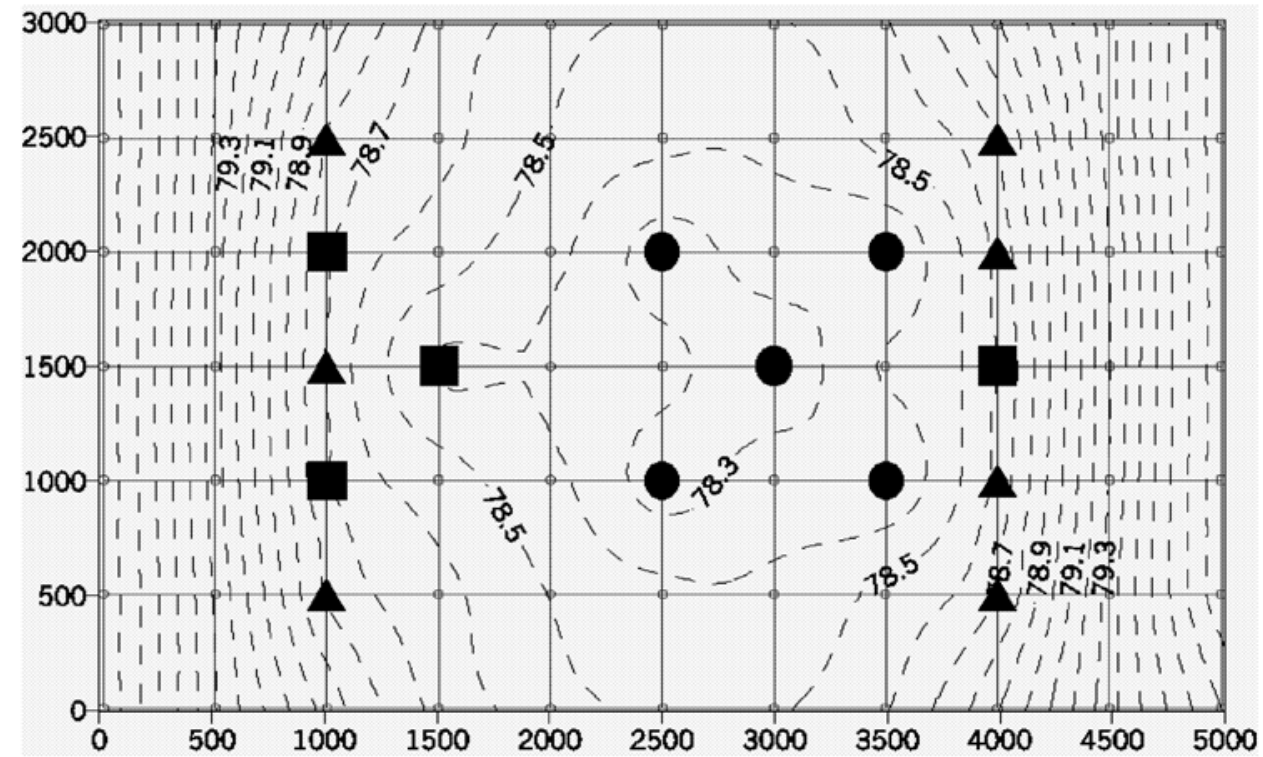

Figure 7. The water level of case 3 (CE and linear increasing demand) at the end of the 15 th year.

value, case 2 has the smallest operation cost. Table 2 shows operation cost for the three cases as $\$ 993,005(100 \%)$, $\$ 1,059,023(107 \%)$, and $\$ 1,123,846(113 \%)$, respectively.

\subsection{Impact of Different Annual Interest Rates on Present Value Cost}

[23] Table 3 summarizes present value cost for cases 1 and 2 under different annual interest rates varying from 8 to $14 \%$. Table 3 indicates that present values of fixed cost for the capacity expansion (CE) model are significantly less than for the non-capacity expansion (NCE) model and that fixed cost present value saving increases with increasing interest rates. The ratio of fixed present value cost of the $\mathrm{CE}$ model to that of the NCE model is $64,58,53$, and $49 \%$ for interest rates at $8,10,12$, and $14 \%$, respectively. The total cost ratio in Table 3 between the two models is $89,86,82$, and $79 \%$ with respect to the same set of interest rate alternatives. Fixed present value cost contributes the most savings to total present value costs. Figure 9 shows the previous results of fixed cost and total cost ratio and it indicates that the cost ratios vary linearly with respect to annual interest rates. On the basis of Figure 9, one can

$\boldsymbol{\Delta}$ : wells for period I, $\boldsymbol{\bullet}$ :wells for period II, $\bullet$ :wells for period III

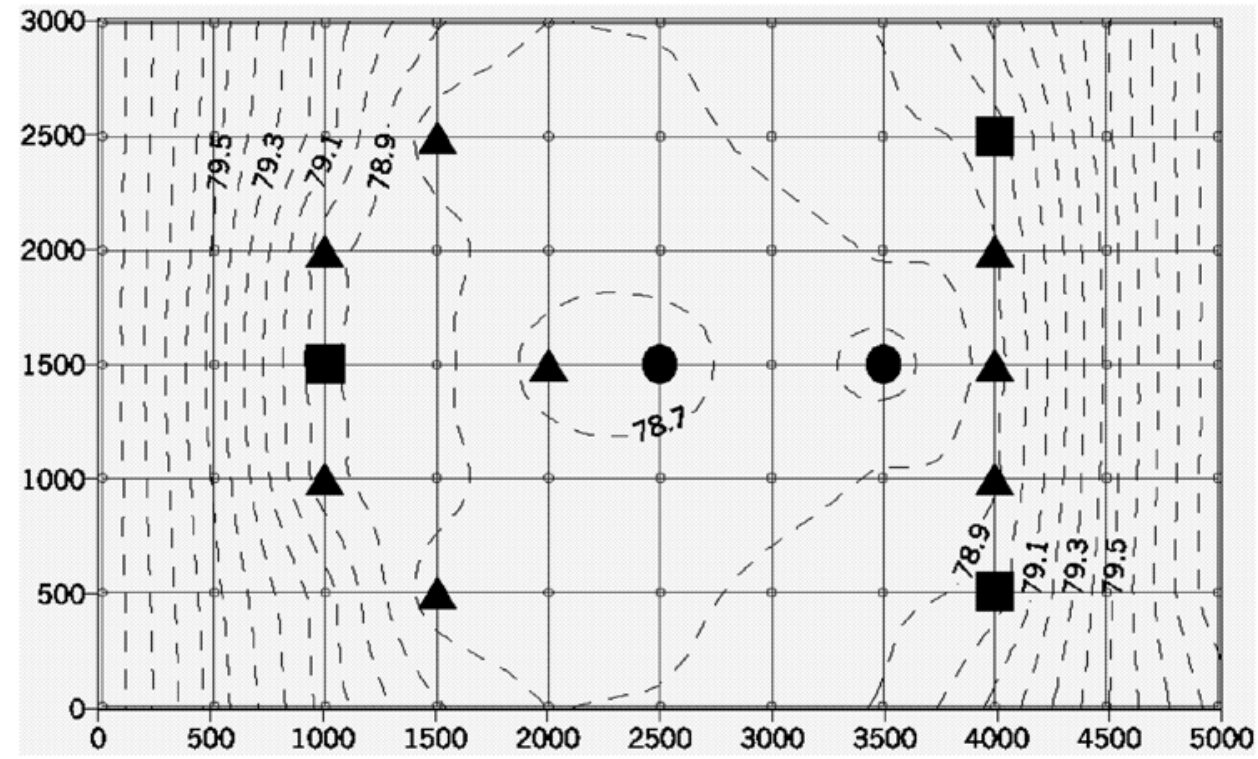

Figure 8. The water level of case 4 (CE and concave increasing demand) at the end of the 15 th year. 


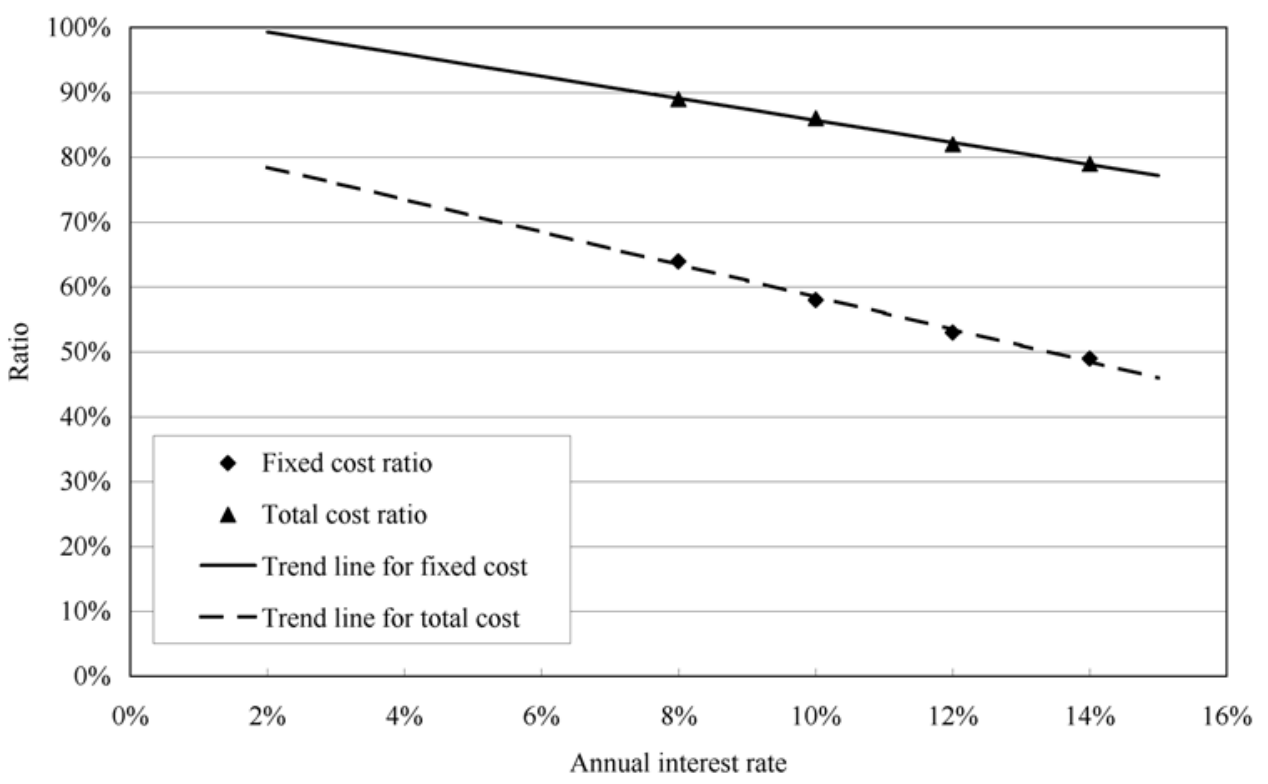

Figure 9. Fixed cost and total cost saving ratio diagram (comparison of case 1 and case 2).

approximately estimate that the ratios of the fixed cost and total cost are higher than 70 and $95 \%$ if the interest rate is less than $5 \%$. As expected, the cost saving for the CE model decreases with lower interest rates.

\section{Discussion}

[24] This section addresses the required computational resources for using the proposed hybrid algorithm to solve a practical field case. Table 4 summarizes the CPU time to solve the four cases. To more objectively estimate the CPU time, this study repeatedly computes each case four times. The average numbers of generations for GA convergences are less than 30 and the average CPU times for the cases considering capacity expansion are nearly $1 \mathrm{~h}$. The computer solving the cases is equipped with Intel ${ }^{\circledR}$ Core $^{\mathrm{TM}} 2$ Quad Processor Q9450 (2.66 GHz CPU) and 4 GB of memory. Table 4 indicates minimal computational loading for these hypothetical cases.

[25] On the basis of CPU time for solving the hypothetical cases, this work estimates the required computational demand when applying the algorithm to a more general field case. When applying the hybrid algorithm, most computational loading derives from the modified CDDP. According to Mansfield [1998], the CPU time for CDDP increases with the order of $O\left(n^{3}\right)$, where $n$ is the number of state variables, and increases with the order of $O(m)$, where $m$ is the number of time steps [Mansfield et al., 1998]. Therefore, rapidly increasing CPU time caused by increased number of state variables is an important computational issue when applying the algorithm to a problem with a large number of state variables. For groundwater system planning cases, the number of state variables equals the number of active nodes, other than the Dirichlet boundary nodes, in the numerical simulation model. Therefore, when applying the proposed algorithm to solve a field case, CPU time approximately increases with the order of $O\left(n d^{3}\right)$ where, $n d$ is the number of active nodes used to simulate the groundwater system. The number of active nodes for hypothetical cases is 63 and the maximum required CPU time is approximately $1 \mathrm{~h}$.

[26] Following the previous discussion, a representative field case with more nodes is used to illustrate the potential increment of computational requirement for a field application, and how the computing time can be managed by parallel computation. For example, assuming a field case with 1260 nodes (20 times of state variables number to the hypothetical cases), the CPU time will be approximately $8000 \mathrm{~h}$ according to the previous discussion for the same computer. Although the CPU time seems very large, the wall clock time can be reduced significantly using parallel computation because the main algorithm of the proposed hybrid algorithm is a GA. Since computing the fitness of each chromosome is independent, the GA efficiently applies parallel computation. If the example field case can be computed using a parallel computer with $100 \mathrm{CPU}$ nodes,

Table 3. Summary of the Cost Present Values Under Different Interest Rates for Cases 1 and 2

\begin{tabular}{lcccc}
\hline & \multicolumn{4}{c}{ Annual Interest Rate } \\
\cline { 2 - 5 } & $8 \%$ & $10 \%$ & $12 \%$ & $14 \%$ \\
\hline \multirow{4}{*}{ Case 2, CE } \\
Fixed cost, FC (USD) & 280,392 & 255,167 & 233,884 & 215,874 \\
Operation cost, OC (USD) & 993,006 & 832,695 & 703,761 & 600,146 \\
Total cost, TC (USD) & $1,273,398$ & $1,087,862$ & 937,644 & 816,020 \\
& Case 1, NE & & \\
Fixed cost, FC (USD) & 440,000 & 440,000 & 440,000 & 440,000 \\
Operation cost, OC (USD) & 992,484 & 831,336 & 702,228 & 597,603 \\
Total cost, TC (USD) & $1,432,484$ & $1,271,336$ & $1,142,228$ & $1,037,603$ \\
Fixed cost, FC (\%) & Costs Ratio, CE/NE & & \\
Operation cost, OC (\%) & 64 & 58 & 53 & 49 \\
Total cost, TC (\%) & 100.05 & 100.16 & 100.22 & 100.43 \\
\hline
\end{tabular}


Table 4. Summary of the Performance Analysis

\begin{tabular}{lccc}
\hline \multicolumn{1}{c}{ Case 1 } & Case 2 & Case 3 & Case 4 \\
\hline & First Test & & \\
Number of GA generation & 21 & 30 & 35 \\
CPU Times (min) & 39.9 & 62.0 & 68.5 \\
& Second Test & & \\
Number of GA generation & 18 & 46 & 17 \\
CPU Times (min) & 34.9 & 94.0 & 35.1 \\
& Third Test & & \\
Number of GA generation & 18 & 18 & 28 \\
CPU Times (min) & 40.5 & 39.3 & 57.7 \\
& Fourth Test & & \\
Number of GA generation & 35 & 20 & 26 \\
CPU Times (min) & 75.6 & 40.5 & 50.0 \\
Number of GA generation & Average & & \\
CPU Times (min) & 23 & 29 & 27 \\
\hline
\end{tabular}

wall clock time will be only $80 \mathrm{~h}$, which is less than 1 week. The estimation is based on each single CPU having the same computing power with that used to solve the hypothetical cases. Using a more powerful CPU and more CPU nodes can possibly reduce wall clock time to within 1 day. In summary, although computational loading of the proposed algorithm increases significantly with problem size (number of nodes), parallel computing and an advanced computing machine can still solve a practical field problem within reasonable wall clock time.

\section{Conclusion}

[27] The proposed approach optimizes minimum total cost, including fixed cost and time-varying operation cost, of a groundwater supply system on unconfined aquifers under a capacity expansion framework. The decision variables of the groundwater network include well locations, installation schedule, and time-varying pumping rates for each well in the network. The optimal capacity expansion problem for groundwater management is a complex mixed nonlinear dynamic problem, since well locations and installation schedule are discrete variables while time-varying pumping rates are continuous. The proposed hybrid algorithm optimizes the capacity expansion problem under reasonable computational resources.

[28] Numerical results indicate that installing the well system under capacity expansion significantly saves fixed cost present value (FC) compared to installing a full capacity system initially. Moreover, saving fixed present value cost increases with interest rates. The proposed algorithm solves problems of various water demand patterns, including convex curve, linear curve, and concave curve, and results demonstrate that the model proposes an adequate system expansion schedule according to water demands.

[29] Acknowledgments. The authors would like to thank the National Science Council of China (Taiwan), which supported this research under contract NSC 95-2621-Z-009-001. We also acknowledge C. T. Hsiao and H. C. Chin for the model programming.

\section{References}

Başağaoğlu, H., and H. Yazicigil (1994), Optimal capacity-expansion planning in multiaquifer systems, J. Water Resour. Plann. Manage., 120, 836-856, doi:10.1061/(ASCE)0733-9496(1994)120:6(836).

Braga, B. P. F., J. G. L. Conejo, L. Becker, and W. W. G. Yeh (1985), Capacity expansion of Sao Paulo water supply, J. Water Resour. Plann. Manage., 111, 238-252, doi:10.1061/(ASCE)0733-9496(1985) 111:2(238).

Chang, L. C., and C. T. Hsiao (2002), Dynamic optimal ground water remediation including fixed and operation costs, Ground Water, 40, 481-490, doi:10.1111/j.1745-6584.2002.tb02532.x.

Chang, L. C., C. A. Shoemaker, and P. L. F. Liu (1992), Optimal timevarying pumping rates for groundwater remediation: Application of a constrained optimal control algorithm, Water Resour. Res., 28, 31573173, doi:10.1029/92WR01685.

Chang, L. C., H. J. Chu, and C. T. Hsiao (2007), Optimal planning of a dynamic pump-treat-inject groundwater remediation system, J. Hydrol., 342, 295-304, doi:10.1016/j.jhydrol.2007.05.030.

Chu, H. J., C. T. Hsiao, and L. C. Chang (2005), Optimal remediation design in groundwater systems by intelligent techniques, in Knowledge-Based Intelligent Information and Engineering Systems, Part 2 Proceedings, Lecture Notes Artif. Intell., vol. 3682, edited by R. Khosla, R. J. Howlett, and L. C. Jain, pp. 628-634, Springer, Berlin.

De Jong, K. (1975), An analysis of the behavior of a class of genetic adaptive systems, Ph.D. thesis, Univ. of Mich., Ann Arbor.

Hsiao, C. T., and L. C. Chang (2002), Dynamic optimal groundwater management with inclusion of fixed costs, J. Water Resour. Plann. Manage., 128, 57-65, doi:10.1061/(ASCE)0733-9496(2002)128:1(57).

Hsiao, C. T., and L. C. Chang (2005), Optimizing remediation of an unconfined aquifer using a hybrid algorithm, Ground Water, 43, 904-915.

Jenkins, M. W., J. R. Lund, R. E. Howitt, A. J. Draper, S. M. Msangi, S. K. Tanaka, R. S. Ritzema, and G. F. Marques (2004), Optimization of California's water supply system: Results and insights, J. Water Resour. Plann. Manage., 130, 271-280, doi:10.1061/(ASCE)07339496(2004)130:4(271).

Jones, L. D., R. Willis, and W. W. G. Yeh (1987), Optimal control of nonlinear groundwater hydraulics using differential dynamic programming, Water Resour. Res., 23,2097-2106, doi:10.1029/ WR023i011p02097.

Mahmoud, M. R. (2006), High dimension dynamic programming model for water resources expansion projects, Eng. Optim., 38, 371-389, doi:10.1080/03052150600593218.

Mansfield, C. M., C. A. Shoemaker, and L. Z. Liao (1998), Utilizing sparsity in time-varying optimal control of aquifer cleanup, J. Water Resour. Plann. Manage., 124, 15-21, doi:10.1061/(ASCE)07339496(1998)124:1(15).

Murray, D. M., and S. J. Yakowitz (1979), Constrained differential dynamic programming and its application to multireservoir control, Water Resour. Res., 15, 1017-1027, doi:10.1029/WR015i005p01017.

Pinder, G. F., and E. O. Frind (1972), Application of Galerkin's procedure to aquifer analysis, Water Resour. Res., 8, 108-120, doi:10.1029/ WR008i001p00108.

Pulido-Velazquez, M., J. Andreu, and A. Sahuquillo (2006), Economic optimization of conjunctive use of surface water and groundwater at the basin scale, J. Water Resour. Plann. Manage., 132, 454-467, doi:10.1061/(ASCE)0733-9496(2006)132:6(454).

Rosegrant, M. W., and X. M. Cai (2002), Global water demand and supply projections part 2. Results and prospects to 2025, Water Int., 27, $170-$ 182, doi:10.1080/02508060208686990.

Voivontas, D., G. Arampatzis, E. Manoli, C. Karavitis, and D. Assimacopoulos (2003), Water supply modeling towards sustainable environmental management in small islands: The case of Paros, Greece, Desalination, 156, 127-135, doi:10.1016/S0011-9164(03)00335-7.

Watkins, D. W., and D. C. McKinney (1998), Decomposition methods for water resources optimization models with fixed costs, Adv. Water Resour., 21, 283-295, doi:10.1016/S0309-1708(96)00061-9.

L.-C. Chang, Y.-W. Chen, and M.-S. Yeh, Department of Civil Engineering, National Chiao Tung University, 1001 Ta Hsueh Road, Hsinchu, 300, Taiwan. (lcchang31938@gmail.com) 